\title{
Solar hard X-ray halo from decaying neutrons
}

\author{
A. L. MacKinnon \\ DACE/Department of Physics and Astronomy, University of Glasgow, Glasgow G12 8QQ, UK \\ e-mail: a.mackinnon@educ.gla.ac.uk \\ Received 31 August 2006 / Accepted 24 October 2006 \\ ABSTRACT

\begin{abstract}
Aims. To quantify the solar X-ray halo resulting from inner bremsstrahlung in beta decay of neutrons generated by cosmic ray bombardment of the solar surface.

Methods. We show analytically how the angular form of this X-ray halo directly reflects the energy distribution of neutrons escaping the Sun. Previous Monte Carlo calculations of solar albedo neutron production are used to normalise an assumed parametric form for the escaping neutron distribution and thus to estimate the halo's intensity. The main remaining assumptions, that neutrons escape radially and that gravitational deceleration may be neglected, affect a negligible fraction of all solar neutrons.

Results. Observations of this halo would have clear diagnostic interest for cosmic ray propagation in the inner heliosphere. Unfortunately it is unlikely to be observable in competition with the cosmic X-ray background.
\end{abstract}

Key words. Sun: X-rays, gamma rays - plasmas - acceleration of particles

\section{Introduction}

Inner bremsstrahlung (IB) is the name given to the electromagnetic radiation that accompanies beta decay of neutrons, or indeed any beta-unstable nuclei. Neutron IB was previously employed by Petrosian \& Ramaty (1972) to comment on the viability of the Steady State cosmology in the light of the observed intensity of the cosmic X-ray background.

Cosmic rays impacting the solar surface will produce a quasi-static population of free neutrons surrounding the Sun (Seckel et al. 1991). This neutron population may be augmented by any ion acceleration accompanying the coronal heating process. Thus, the Sun will be surrounded by a faint, extended X-ray glow produced by IB emission as these neutrons decay. The existence of this halo should not be forgotten in discussing signatures of e.g. decaying WIMPs (Weakly Interacting, Massive Particles - DiLella \& Zioutas 2003; Zioutas et al. 2004). It may also serve as a diagnostic for the ion population normally impinging on the solar surface.

The mean lifetime of a free neutron is about $15 \mathrm{~min}$. Solar neutrons around $\mathrm{a} \mathrm{GeV}$ in energy will thus extend over a region of radius $\sim 2 \mathrm{AU}$. Much more energetic neutrons will be found throughout a greater region because of their relativistically dilated lifetimes. Neutrons of significantly lower energies will travel lesser distances before they decay. Thus, the angular dependence of the intensity of this X-ray halo reflects the energy distribution of neutrons escaping from the Sun. Its extent distinguishes neutron IB from other cosmic ray secondary processes (e.g. via pion production), which will be observed only from the immediate vicinity of the solar surface.

Properties of IB are calculated as a corollary to the Fermi theory of beta decay (Knipp \& Uhlenbeck 1936; Bloch 1936). IB radiation is more intense than the subsequent bremsstrahlung yield of the beta-decay produced electrons - we get $\sim \frac{1}{137}$ photons per decay, as opposed to the typically $10^{-5}$ photons per electron slowing down in a thick target (e.g. Koch \& Motz 1959).
Neutrons decaying at rest produce a very hard photon spectrum with an upper cutoff at $781 \mathrm{keV}$.

We describe this X-ray spectrum in more detail in Sect. 2. In Sect. 3 we show how the angular form of the IB X-ray halo reflects the energy distribution of neutrons escaping from the Sun. Section 4 leans on the previous calculation of Seckel et al. (1991) to estimate the intensity of the X-ray halo resulting from neutrons produced by cosmic rays impacting the solar surface. This halo appears not to be competitive with the cosmic X-ray background. A concluding Section considers factors that might enhance the IB flux to the level of observability and discusses further the diagnostic potential of this new component of the solar X-ray emission.

\section{Photon spectrum of inner bremsstrahlung}

The calculation of the neutron beta decay photon spectrum, carried out by Bloch (1936) and by Knipp \& Uhlenbeck (1936), is summarised in Petrosian \& Ramaty (1972). The photon flux goes identically to zero at $781 \mathrm{keV}$, the maximum energy of electrons emitted in neutron beta decay. The assumptions in this theoretical spectrum break down and it suffers a mild infrared catastrophe as photon energy $\epsilon \rightarrow 0$ (finite energy flux, but diverging photon number flux - Bloch 1936) but it is accurate in the X-ray photon energy range. The photon spectrum $i(\epsilon)$ (photons $\mathrm{keV}^{-1}$ neutron ${ }^{-1}$ ) produced by neutrons decaying at rest is shown in Fig. 1 and is approximated to within 10\%, for photon energies below $650 \mathrm{keV}$, by this functional form:

$i(\epsilon)= \begin{cases}\frac{A}{\epsilon} \exp \left(-\epsilon / \epsilon_{0}\right) & \left(\epsilon<2.3 \epsilon_{0}\right) \\ \frac{A}{\epsilon} \exp \left(4-2.74 \epsilon / \epsilon_{0}\right) & \left(\epsilon>2.3 \epsilon_{0}\right)\end{cases}$

where $\epsilon$ is measured in $\mathrm{keV}, \epsilon_{0}=184 \mathrm{keV}$ and $A=1.5 \times$ $10^{-3}$ neutron $^{-1}$.

This is a very hard spectrum, roughly like $\epsilon^{-1}$ in the 10-100 keV energy range (Fig. 1). It represents the neutron 


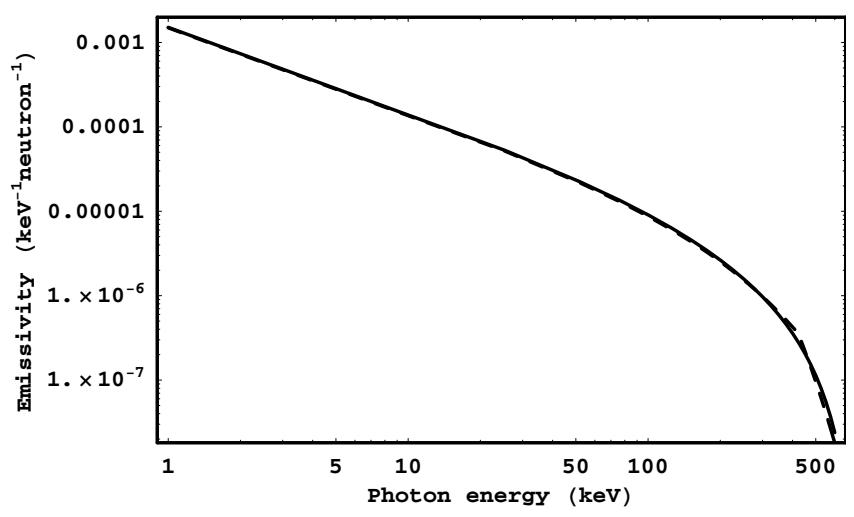

Fig. 1. Inner bremsstrahlung photon spectrum per neutron calculated numerically from the expressions given in Petrosian \& Ramaty (1972) (solid line). Also shown for comparison is the approximate fit (1) (dashed line) but the difference between the two curves is very small in this energy range, mostly $\ll 10 \%$, so that they can barely be discriminated visually.

rest frame photon spectrum, and should in principle be transformed into the observer's frame. As long as the distribution of neutrons escaping from the Sun declines with energy (as it does for instance in the case of the Earth, e.g. Hess et al. 1959; Gordon et al. 2004), the majority of neutrons present along the line of sight within $90^{\circ}$ of the Earth-Sun direction will be non-relativistic; see Sect. 3.1 below. Thus, we may ignore this complication for present purposes so that the photon fluence from a fixed, initial population of neutrons is just given by the total number of neutrons multiplied by the photon fluence $i(\epsilon)$ per neutron.

\section{Information content of the IB X-ray halo}

\subsection{Decay of free neutrons}

The lifetime $\tau$ of the free neutron is still uncertain to some degree. In what follows we adopt the current value of $886 \mathrm{~s}$ (Yao et al. 2006). If neutrons of kinetic energy $E$ are produced at the solar surface and emitted radially, the probability of survival at a distance $r$ from the solar centre is

$\exp \left\{\frac{R_{\odot}-r}{\beta \gamma c \tau}\right\}$.

The symbols have their usual meanings, i.e. $c$ is the speed of light, $R_{\odot}$ is the solar radius, $\gamma=1+E / m_{\mathrm{n}} c^{2}, m_{\mathrm{n}}$ is the neutron rest mass and $\beta^{2}=1-\gamma^{-2}$. We suppose, with negligible error for what follows, that $E \gg 2 \mathrm{keV}$, the gravitational potential energy of a neutron near the solar surface.

The behaviour of the survival probability becomes more transparent for non-relativistic neutrons. In this case it is given by

$\exp \left\{-\left(\frac{E_{*}(r)}{E}\right)^{1 / 2}\right\}$.

Here $E_{*}(r)$ is the energy of neutron that takes a time $\tau$ to travel from $R_{\odot}$ to distance $r$ :

$E_{*}(r)=\frac{m_{\mathrm{n}}^{2}}{2}\left(\frac{r-R_{\odot}}{\tau}\right)^{2}$.

Numerically, $E_{*}=E_{D} r_{\mathrm{AU}}^{2} \mathrm{MeV}$ when $r_{\mathrm{AU}}$ denotes the value of $r$ in units of AU, $E_{D}(=150 \mathrm{MeV})$ is the energy of neutron that

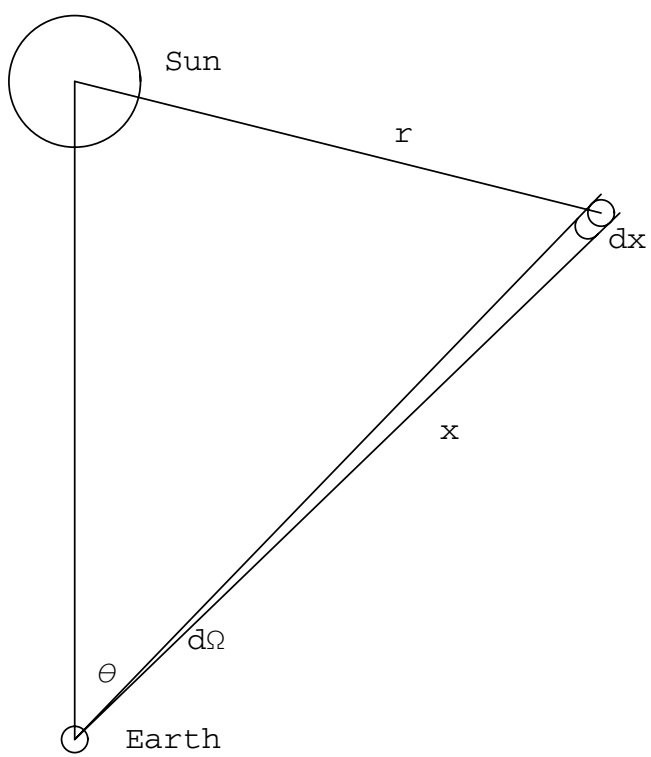

Fig. 2. Geometry for calculation of X-ray halo brightness as a function of angle to the Earth-Sun direction.

travels $1 \mathrm{AU}$ in a lifetime and we neglect $R_{\odot}$ compared with $r$. At distance $r$ there will be few neutrons present with energies much below $E_{*}$. At $1 \mathrm{AU}$ from the Sun, non- to mildly-relativistic neutrons will dominate the population looking in any direction so that the IB X-ray flux will be separable in energy and angle.

\subsection{Neutron distribution far from the Sun}

Suppose that the Sun is bombarded at a steady rate by cosmic rays and that the resulting neutron distribution about the Sun is spherically symmetric. Let $n(E, r) \mathrm{d} E$ be the density of neutrons $\left(\mathrm{cm}^{-3}\right)$ with kinetic energies between $E$ and $E+\mathrm{d} E$ at distance $r$. Let the distribution at the solar surface be $f(E)=$ $n\left(E, R_{\odot}\right)$. We continue to assume that all neutrons move radially outward and to neglect gravitational deceleration, discussing the consequences of these assumptions below (Sect. 4.3). At any distance $r>R_{\odot}, n$ is given by

$n(E, r)=f(E)\left(\frac{R_{\odot}}{r}\right)^{2} \mathrm{e}^{\left(R_{\odot}-r\right) /(\beta \gamma c \tau)}$.

Most of the time the exponential factor may be rewritten assuming non-relativistic neutrons and neglecting $R_{\odot}$ compared to $r$, as above.

\subsection{Angular form of IB flux}

Consider now the total IB yield from within a cone of solid angle $\mathrm{d} \Omega$, centred on a line of sight at an angle $\theta$ to the SunEarth direction (Fig. 2). We assume that the Sun's neutron halo is spherically symmetric, so that IB radiation viewed from Earth is azimuthally symmetric about the Sun-Earth line. Because almost all neutrons are nonrelativistic, IB radiation is isotropic and the volume photon emissivity $\left(\mathrm{keV}^{-1} \mathrm{sterad}^{-1} \mathrm{~cm}^{-3} \mathrm{~s}^{-1}\right)$ at position $r$ and photon energy $\epsilon$ is given by

$j(r, \epsilon)=\frac{i(\epsilon)}{4 \pi \tau} \int_{0}^{\infty} n(E, r) \mathrm{d} E$

A detector of unit area at distance $x$ subtends a solid angle of $1 / x^{2}$. The volume element subtending solid angle $\mathrm{d} \Omega$ at 
distance $x$ from Earth, at angle $\theta$ to the Earth-Sun direction (Fig. 2) has volume $x^{2} \mathrm{~d} \Omega \mathrm{d} x$ and thus contributes

$j(x, \theta, \epsilon) \mathrm{d} x \mathrm{~d} \Omega$

to the photon flux $\left(\mathrm{keV}^{-1} \mathrm{~cm}^{-2}\right.$ sterad $\left.^{-1} \mathrm{~s}^{-1}\right)$ observed from within solid angle $\mathrm{d} \Omega$ at distance $x$.

Immediately, we see that the IB spectrum at Earth $I(\epsilon, \theta)$ (photons $\mathrm{keV}^{-1} \mathrm{~s}^{-1} \mathrm{~cm}^{-2}$ sterad $^{-1}$ ), at photon energy $\epsilon$ and angle $\theta$ to the Earth-Sun distance (Fig. 2) is

$I(\epsilon, \theta)=\frac{i(\epsilon)}{4 \pi \tau} \int_{0}^{\infty} \int_{0}^{\infty} n(r(x, \theta), E) \mathrm{d} E \mathrm{~d} x$

with $n(r)$ and $i(\epsilon)$ given by Eqs. (3) and (1), respectively,

$r^{2}=x^{2}+D^{2}-2 x D \cos \theta$

and $D=1$ AU.

Using (3) in (4) we rewrite this in terms of $f(E)$ :

$I(\epsilon, \theta)=i(\epsilon) \frac{R_{\odot}^{2}}{4 \pi \tau} \int_{0}^{\infty} f(E) K(E, \theta) \mathrm{d} E$

where

$K(E, \theta)=\int_{0}^{\infty} r^{-2} \exp \left\{\frac{R_{\odot}-r}{\beta \gamma c \tau}\right\} \mathrm{d} x$

and the dependences (5) in $r(x, \theta)$ are to be understood in the integrand.

The observed photon spectrum $I(\epsilon, \theta)$ will be separable in $\epsilon$ and $\theta$. Its $\theta$-dependence is determined by an integral over the neutron energy distribution $f(E)$ at the Sun. Measurements of this $\theta$-dependence might allow us to deduce $f(E)$ via the usual regularization techniques (e.g. Craig \& Brown 1986) applied to Eqs. (6) and (7), or via forward fitting of parametric models. In turn, then, we might comment on the energy distribution of cosmic rays arriving at the Sun and their transport in the solar atmosphere.

Some further analytical development is possible, which might aid in the application of standard numerical techniques. In the Appendix we give an approximate form for $K(E, \theta)$, valid roughly for $5^{\circ}<\theta<75^{\circ}$. Using this in (6) we find

$\frac{I(\epsilon, \theta)}{i(\epsilon)}=\frac{R_{\odot}^{2} E_{D}}{\sqrt{2 \pi} \tau D \sin ^{3 / 2} \theta} \mathcal{L}(g, \sin \theta)$

where $\mathcal{L}(h, p)$ is the Laplace transform of the function $h$ with respect to the parameter $p$, and

$g(s)=s^{-7 / 2} f\left(E_{D} s^{-2}\right)$.

The angle dependence of the X-ray flux is given approximately by the Laplace transform of the initial neutron energy distribution at the Sun.

\section{Likely intensity of X-ray halo}

\subsection{Previous calculation of albedo neutron production}

Seckel et al. (1991) carried out calculations aimed at predicting observable consequences of cosmic ray bombardment of the solar surface. They treated cosmic ray transport in interplanetary turbulence and in active region magnetic fields near the solar surface, combining this with a Monte Carlo treatment of nuclear processes in the solar atmosphere to calculate yields of various secondaries. Their adopted model of solar surface magnetic features was considered appropriate to Quiet Sun or solar minimum conditions. They did not discuss IB X-rays or in situ detection of neutrons near the Sun, and calculated only the expected integrated flux of neutrons above $100 \mathrm{MeV}$ at 1 AU: $2.3 \times 10^{-8} \mathrm{~cm}^{-2} \mathrm{~s}^{-1}$. Direct detection of this flux and discrimination from other, background signals at Earth would be challenging.

\subsection{Solar albedo neutron energy distribution}

Below we use Seckel et al.'s predicted neutron flux as the starting point for an estimate of IB X-ray intensity. In the absence of further Monte Carlo calculations we may use this number with an assumed form for $f(E)$ to estimate neutron density near the Sun and thus IB X-ray flux.

Calculations of escaping neutron distributions in flares (e.g. Hua \& Lingenfelter 1987; Hua et al. 2002) have certain characteristic features. At energies of $10 \mathrm{~s}$ of $\mathrm{MeV}$ and above, the neutron energy distribution mirrors the incident, primary ion energy distribution. At lower energies it becomes almost energyindependent, dominated by the scattering and thermalisation processes inevitably implicated in neutron escape from the Sun, as well as by the behaviour of the production cross-sections near threshold.

Thus, the energy distribution of escaping neutrons may be modelled with the functional form

$f(E)=(\delta-1)\left(\frac{n_{\odot}}{E_{0}}\right)\left(1+\frac{E}{E_{0}}\right)^{-\delta}$.

Here $n_{\odot}$ is the total neutron density at the solar surface. The energy power-law index $\delta$ is chosen to mirror the energy distribution of cosmic rays at higher energies i.e. $\delta=3.3$ (e.g. Longair 1981) and we adopt the representative value $E_{0}=10 \mathrm{MeV}$ (cf. Figs. 6 and 7 of Hua et al. 2002).

Terrestrial atmospheric neutrons measured at sea level or at high altitude (Hess et al. 1959) offer another guide to the possible form of the solar neutron energy distribution, albeit one dominated by cosmic ray interaction with heavier species. This distribution is harder in the $>100 \mathrm{MeV}$ energy range than our form (9) but steepens again below about $20 \mathrm{MeV}$ (Gordon et al. 2004). Below we also comment on the consequences of adopting this distribution as a guide.

\subsection{Albedo neutron density near the Sun and IB $X$-ray flux}

We can use the predicted neutron flux from Seckel et al. (1991) and the guessed energy distribution (9) to estimate the neutron density near the Sun. First of all we fix $n_{\odot}$ by requiring that Eqs. (3) and (9) together imply the same flux at $1 \mathrm{AU}$ of $>100 \mathrm{MeV}$ neutrons predicted by Seckel et al. (1991). We find $n_{\odot}=4 \times 10^{-11} \mathrm{~cm}^{-3}$.

We can now make some preliminary rough estimates of the form and intensity of the neutron IB halo. Neutron numbers fall off rapidly above $E_{0}=10 \mathrm{MeV}$, while lower energy neutrons decay closer to the Sun. Thus, the characteristic size of the IB halo will be given by the distance travelled by $10 \mathrm{MeV}$ neutrons in a time $\tau$, about $70 R_{\odot}$. The angular radius of the halo will be

$\tan ^{-1}\left(\frac{70 R_{\odot}}{\mathrm{AU}}\right)=18^{\circ}$.

There will be $\simeq 2 \times 10^{28}$ neutrons in total within a Sun-centred volume of radius $70 R_{\odot}$, decaying at a rate of $2.3 \times 10^{25} \mathrm{~s}^{-1}$. 


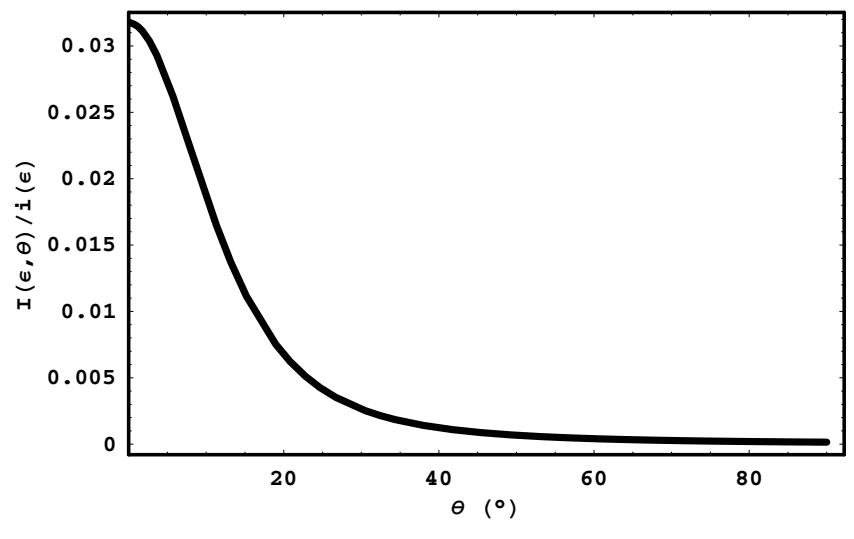

Fig. 3. $I(\epsilon, \theta) / i(\epsilon)$, as a function of the angle $\theta$ between the line of sight and the Earth-Sun direction.

As noted above, IB involves the emission of about $1 / 137$ photons per decay, so we estimate a total photon flux of $6 \times 10^{-5} \mathrm{~cm}^{-2} \mathrm{~s}^{-1}$ from the whole of this volume at Earth. If this were a point source, not too close to the Sun, it would be easily detectable. It is for instance roughly three orders of magnitude brighter than the faintest point sources detected, in the $2-10 \mathrm{keV}$ range, in recent studies of the cosmic X-ray background (Moretti et al. 2003). Its large angular extent will make it much harder to observe, however, as we see below.

As a more precise illustration, Fig. 3 shows the angular part of the IB X-ray flux as a function of angle $\theta$ to the EarthSun direction, using $\delta=3.3, E_{0}=10 \mathrm{MeV}$ and fixing $n_{\odot}$ as above. Values shown in this figure need only be multiplied by the value of $i(\epsilon)$ at a particular value of $\epsilon$, or its integral over a given photon energy range, to yield a photon flux $I(\epsilon)$ (photons $\mathrm{cm}^{-2} \mathrm{keV}^{-1}$ sterad $^{-1} \mathrm{~s}^{-1}$ ).

Figure 3 was calculated using the exact expressions (6) and (7). Apart from the adoption of the energy distribution (9), the main assumptions are that all neutrons travel radially, and that gravitational deceleration may be neglected. The former assumption minimises neutron travel times to any given location, and thus sets an upper limit on the IB flux from any direction. Its relaxation will be most important for neutrons that travel $R_{\odot}$ or less in a time comparable to $\tau$, i.e. for neutron energies of about $4 \mathrm{keV}$ or less. As already mentioned, neutrons of $2 \mathrm{keV}$ or less do not actually escape so these will be present near the Sun in greater numbers than estimated here. $10 \mathrm{keV}$ is thus a rough upper limit to the neutron energy at which both these effects become important. With the form (9), such neutrons amount to just $0.2 \%$ of the total population. A different assumption about the angular distribution of neutrons would affect Fig. 3 negligibly, as would the gravitational trapping of low energy neutrons near the Sun.

Gordon et al. (2004) give an analytical expression, valid in the range $0.1-10000 \mathrm{MeV}$, representing their experimentally measured results for the terrestrial neutron energy distribution. We repeated the above exercise using this energy distribution. The X-ray flux then falls off much more steeply with angle than shown in Fig. 3, dropping by an order of magnitude in just $2^{\circ}$. This is because this distribution continues to increase to lower neutron energies, as opposed to the energy-independence characterising the low-energy behaviour of (9). However, the terrestrial neutron distribution is much flatter in the $10-100 \mathrm{MeV}$ range than (9), especially with $\delta=3.3$, so total numbers, fixed by the Seckel et al. 1 AU result, are much smaller and the peak intensity of the X-ray halo is smaller by two orders of magnitude.

\subsection{Cosmic X-ray background}

We must compare this predicted X-ray flux with the general (cosmic) X-ray background flux which has a value of $2 \times$ $10^{-11} \mathrm{erg} \mathrm{s}^{-1} \mathrm{~cm}^{-2} \mathrm{deg}^{-2}$ integrated over the $2-10 \mathrm{keV}$ photon energy range (McCammon \& Sanders 1990; Moretti et al. 2003). To compare the Sun's IB halo with this value, we simply multiply the values in Fig. 3 by

$\int_{2}^{10} \epsilon i(\epsilon) \mathrm{d} \epsilon$

i.e. $1.86 \times 10^{-11}\left(\mathrm{erg}\right.$ neutron $\left.{ }^{-1}\right)$. Near the Sun, then, the IB flux will approach $1.6 \times 10^{-16} \mathrm{erg} \mathrm{s}^{-1} \mathrm{~cm}^{-2} \mathrm{deg}^{-2}$, some five orders of magnitude below the general cosmic X-ray background. In this low energy range there may also be competition from thermal, coronal emission, but the comparison remains similarly unfavourable at photon energies $>100 \mathrm{keV}$ (as given in e.g. Kinzer et al. 1997). Thus, it seems unlikely that this X-ray flux will be observable in competition with the cosmic X-ray background, at least as long as we consider only neutrons resulting from bombarding galactic cosmic rays.

\section{Discussion}

The Sun's IB halo, a previously undiscussed component of solar X-radiation, posesses significant potential as a diagnostic of the fast ion distribution impinging on the solar surface. As we have seen here, its angular form directly reflects the energy distribution of neutrons escaping from the Sun. Our estimate of its intensity suggests it will be too faint to observe, however, at least if produced only by cosmic rays. We list some factors that might work in the direction of optimism.

First, any Quiet Sun ion acceleration, possibly entailed in the coronal heating process, will enhance the escaping neutron population and the IB halo intensity. We have to note the existence of observational upper limits to the Quiet Sun photon flux in the $2.223 \mathrm{MeV}$ neutron capture line, ruling out $>10 \mathrm{MeV}$ ions as a major energy transport mechanism in coronal heating (Harris et al. 1992) and incidentally placing limits on the likely Quiet Sun neutron population.

The work of Seckel et al. (1991) plays a central role in the estimate of IB intensity so we should note some of its assumptions. It employs a specific model for small-scale magnetic fields which should be re-examined, particularly since potential extrapolations from magnetogram data are now available routinely (e.g. Schrijver \& DeRosa 2003). At solar minimum, as the Sun's magnetic polarity reverses, there may be times when cosmic rays arrive much more easily at the solar surface (see Moraal et al. 2005) and the escaping neutron population would be temporarily enhanced at these times. Seckel et al. neglect incident and target species heavier than ${ }^{4} \mathrm{He}$, even although these can make a non-negligible contribution to neutron production in flares (Hua \& Lingenfelter 1987; Hua et al. 2002). We note that the updated treatment of reaction kinematics used in Hua et al. (2002) accounts for a $50 \%$ increase in neutron yields over the earlier treatment of Hua \& Lingenfelter (1987).

Adopting the terrestrial neutron energy distribution as an alternative to (9) resulted in a much fainter X-ray halo, fading much more rapidly with angle from the solar direction. Measurements (Gordon et al. 2004) represent the neutron energy distribution within the terrestrial atmosphere, not the distribution of escaping neutrons. In the flare calculations of Hua et al. (2002), few lower energy, multiply scattered neutrons 
escape. The assumptions made there about the propagation of accelerated ions in flare loops may not be correct for discussing the impact of galactic cosmic rays on the solar surface, however. Together with the factors mentioned in the previous paragraph, this highlights the need for further calculations of cosmic ray neutron production in the spirit of Seckel et al. (1991), concentrating particularly on the angular and energy distribution of lower energy neutrons near the Sun.

Seckel et al. (1991) commented that the neutron flux at Earth following a flare was many orders of magnitude greater than their predicted, cosmic-ray produced neutron flux. The neutrons escaping into space following a flare (Chupp et al. 1987) will also radiate via IB, though the angular distribution of this radiation will have a rapidly time-varying form of quite different significance to that discussed here. A preliminary estimate suggests that the neutrons accompanying a large flare, e.g. as described by Chupp et al. (1987) will produce an IB photon flux comparable with the background signal experienced in the RHESSI (Lin et al. 2002) mission, thus potentially close to observable. This component of flare X-radiation will be discussed elsewhere.

Finally we note that neutron detectors planned for inner heliosphere space missions (e.g. Bravar et al. 2005) should be able to directly detect the quasi-static escaping neutron population: the very modest density found in Sect. 4.3 still corresponds to a flux near the Sun of order 1 neutron $\mathrm{cm}^{-2} \mathrm{~s}^{-1}$. Even if IB X-radiation proves unobservable from $1 \mathrm{AU}$, the diagnostic possibilities of this neutron population, directly detected, should still be exploitable.

Acknowledgements. I first learnt about inner bremsstrahlung from the late Reuven Ramaty, as a summer visitor to NASA Goddard Space Flight Center in 1984. I thank Hugh Hudson for useful comments and discussions. The referee, Brian Dennis, also made helpful comments. Solar physics research in Glasgow is supported by a PPARC Rolling Grant. This research has made use of NASA's Astrophysics Data System.

\section{Appendix A: Approximate kernel for angular distribution}

Making the change of variable $u=x / D$, neglecting $R_{\odot}$ compared to $x$ and working in the non-relativistic regime, (7) may be written

$$
\begin{aligned}
K(E, \theta)= & \frac{1}{D} \int_{0}^{\infty} \frac{1}{u^{2}+1-2 u \cos \theta} \\
& \times \exp \left\{-\left(\frac{E_{D}}{E}\right)^{1 / 2}\left(u^{2}+1-2 u \cos \theta\right)^{1 / 2}\right\} \mathrm{d} u
\end{aligned}
$$

where $E_{D}$ is as defined in Sect. 3.1. The integrand here has a maximum at $u=\cos \theta$, at the point along the line of sight nearest to the Sun. An integrand with a maximum in the argument of an exponential is readily approximated by the method of steepest descents. Writing all of the integrand as an exponential, Taylor expanding the argument of this exponential about $u=\cos \theta$, keeping terms to second order, extending the limits of integration to $(-\infty, \infty)$, and finally evaluating the resulting integral, we get

$$
\begin{aligned}
K(E, \theta) \approx & \frac{\sqrt{\pi}}{D \sin \theta}\left(1+\frac{1}{2}\left(\frac{E_{D}}{E}\right)^{1 / 2} \sin \theta\right)^{-1 / 2} \\
& \times \exp \left\{-\left(\frac{E_{D}}{E}\right)^{1 / 2} \sin \theta\right\}
\end{aligned}
$$

We find numerically that this gives an acceptable approximation, to within a few percent, for $K(E, \theta)$ between about $5^{\circ}$ and $75^{\circ}$ and $E<10 \mathrm{MeV}$. In this range of energy and angle we can go slightly further and use

$$
\left(1+\frac{1}{2}\left(\frac{E_{D}}{E}\right)^{1 / 2} \sin \theta\right)^{-1 / 2} \approx \sqrt{2}\left(\frac{E}{E_{D}}\right)^{1 / 4} \sin ^{-1 / 2} \theta
$$

in (A.2) to proceed fairly rapidly to the result (8).

\section{References}

Bloch, F. 1936, Phys. Rev., 50, 272

Bravar, U., Bruillard, P. J., Flueckiger, E. O., et al. 2005, in Solar Physics and Space Weather Instrumentation, ed. S. Fineschi, \& R. A. Viereck, Proc. SPIE, 5901,141

Chupp, E. L., Debrunner, H., Flueckiger, E., et al. 1987, ApJ, 318, 913

Craig, I. J. D., \& Brown, J. C. 1986, Inverse Problems in Astronomy (Bristol and Boston: Adam Hilger Ltd.)

DiLella, L., \& Zioutas, K. 2003, Astropart. Phys., 19, 145

Gordon, M. S., Goldhagen, P., Rodbell, K. P., et al. 2004, IEEE Trans. Nucl. Sci., 51,3427

Harris, M. J., Share, G. H., Beall, J. H., \& Murphy, R. J. 1992, Sol. Phys., 142, 171

Hess, W. N., Patterson, H. W., \& Wallace, R. 1959, Phys. Rev., 116, 445

Hua, X., Kozlovsky, B., Lingenfelter, R. E., Ramaty, R., \& Stupp, A. 2002, ApJS, 140, 563

Hua, X.-M., \& Lingenfelter, R. E. 1987, ApJ, 323, 779

Kinzer, R. L., Jung, G. V., Gruber, D. E., Matteson, J. L., \& Peterson, L. E. 1997, ApJ, 475, 361

Knipp, J. K., \& Uhlenbeck, G. E. 1936, Physica, 3, 425

Koch, H. W., \& Motz, J. W. 1959, Rev. Mod. Phys., 31, 920

Lin, R. P., Dennis, B. R., Hurford, G. J., et al. 2002, Sol. Phys., 210, 3

Longair, M. S. 1981, High energy astrophysics (Cambridge and New York: Cambridge University Press)

McCammon, D., \& Sanders, W. T. 1990, ARA\&A, 28, 657

Moraal, H., Caballero-Lopez, R. A., McCracken, K. G., \& Humble, J. E. 2005, ApJ, 629, 556

Moretti, A., Campana, S., Lazzati, D., \& Tagliaferri, G. 2003, ApJ, 588, 696

Petrosian, V., \& Ramaty, R. 1972, ApJ, 173, L83

Schrijver, C. J., \& DeRosa, M. L. 2003, Sol. Phys., 212, 165

Seckel, D., Stanev, T., \& Gaisser, T. K. 1991, ApJ, 382, 652

Yao, W.-M., Amsler, C., Asner, D., et al. 2006, J. Phys. G, 33, 1

Zioutas, K., Dennerl, K., DiLella, L., et al. 2004, ApJ, 607, 575 